\title{
Structural Models for Coupled Electricity Markets
}

\author{
Michael Kustermann \\ Universität Duisburg-Essen \\ Universitätsstrasse 12
}

45141 Essen

\author{
Prof. Dr. Rüdiger Kiesel \\ Universität Duisburg-Essen \\ Universitätsstrasse 12 \\ 45141 Essen
}

October 31, 2014

\begin{abstract}
One of the major changes in european electricity markets is - besides the increasing share of renewable infeed - the fact that previously independent market areas have been connected. Day-Ahead auctions are no longer done separately and available interconnector capacity are not always auctioned independently from electricity. Instead, interconnector capacity is implicitely auctioned in the Day-Ahead auction of electricity such that price differences between market areas are minimized, respectively overall wellfare is maximized. The latest cornerstone in this evolution of the european electricity market is the so called North Western European Market Coupling (NWE) which is online since February 4th, 2014.

For market participants, such a change in the structure of the market naturally leads to the question of how to model prices in the affected market areas. Particularly, if positions in more than one market area exist, it becomes crucial for risk- and portfoliomanagement to model electricity prices in all areas consistently in one integrated framework.

The model we present extends the class of structural or hybrid models which were introduced by Carmona et al. (2013) and Aid et al. (2013) to a multi market framework. We derive analytical formulae for the distribution of spot prices, for futures prices and even for plain vanilla options.
\end{abstract}

\section{Introduction}

Since the deregulation of the european electricity markets, modeling price dynamics of electricity became an important topic for market participants. Changes in the structure of the markets lead to changes in price behaviour and thus induced the requirement to regularly update and revise existing pricing models. Most of the existing models can be classified in three classes: reduced form models, fundamental models and hybrid models.

Reduced form models are models which try to model the electricity price directly as a stochastic processs. This approach is widely used in equity and interest rate markets.

Fundamental models rely on the fact that electricity can not be stored and thus, has to be produced at the same time it is consumed. Given all information about all costs of all power 
plants in a system, all technical restrictions and a given demand pattern, it is then (theoretically) possible, to determine the cost-optimal production scedule for the entire system. Shadow prices for electricity arise as dual variables in such an optimization problem.

Hybrid models try to combine the advantages of both models. As demand is well predictable with only little stochastic distortion and the supply side is well understood, Carmona and Coulon (2014) argue that valuation of electricity should be done by equilibrium arguments matching supply and demand. This is the core idea behind hybrid models. Not the price itself is modeled as stochastic process, but the driving factors which influence supply and demand. The price of electricity itself is then determined endogenously.

Reduced form models are widely used at trading desks, mainly to value derivatives on futures. Fundamental models are preferred for long term forecasting of price developements and long term investment planning. Hybrid models are relatively new, mathematically challenging and thus not that widely used so far. Their main advantage is that they are easily adaptable to changing market structures and are able to capture the complicated correlation structures between fuels prices and electricity pries (see Carmona and Coulon (2014)).

\section{Central Western European Market Coupling}

To motivate the model, we analyze the price dynamics of Day-Ahead prices in Germany and France before and after the Central Western European Market Coupling (CWE) which was implemented on November 9th, 2010. CWE couples the Day-Ahead markets of Germany, France, Belgium and the Netherlands. Figure 1 shows the geographical area of CWE. Figure 2 shows in the upper plot French hourly Day-Ahead electricity prices from January 1st, 2010 to December 31th, 2011. The lower plot shows the corresponding prices in Germany. November 9th, 2010 is marked in red. Visually, there is no indication for a strong structural break in the markets. Neither the overall price level nor the variability of prices seem to have changed due to the introduction of CWE Market Coupling. However, looking at the scatter plot of hourly German prices versus hourly French prices as it is done in Figure 3 reveals the new market dynamics. The upper plot shows a scatter plot of German (y-axis) versus French (x-axis) prices in Hour 24 before the introduction of CWE, the lower plot shows the same scatter plot after the implementation of CWE. In the upper plot, prices seem so be loosely correlated, higher prices in France indicate higher prices in Germany and vice versa, but correlation is not very high with values between 0.33 in hour 03 and 0.56 in hour 19. On the other hand, after market coupling, German and French prices coincide exactly in about $70 \%$ of the hours. Only in $30 \%$ of the hours, price differentials could be observed.

\section{A Hybrid Model for Coupled Electricity Markets}

We define the model on a complete probability space $(\Omega, \mathcal{F}, \mathbb{P})$. We model $n$ market areas (countries) in which electricity is produced using $m$ different fuels. The risk factors driving the load processes in each country are given by the n-dimensional Brownian Motion

$$
W_{t}^{D}=\left(W_{t}^{1}, \ldots, W_{t}^{n}\right)
$$




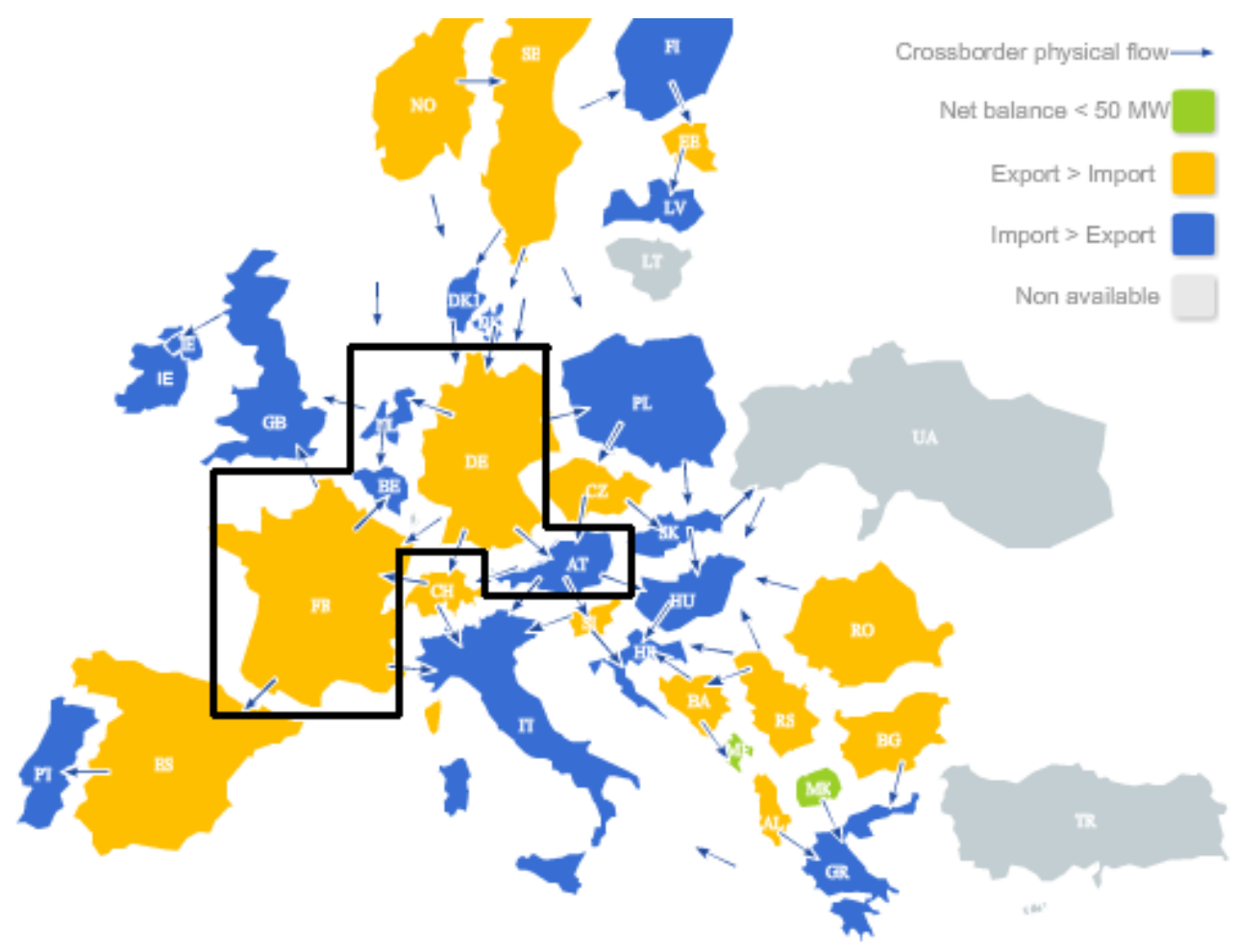

Figure 1: CWE Region
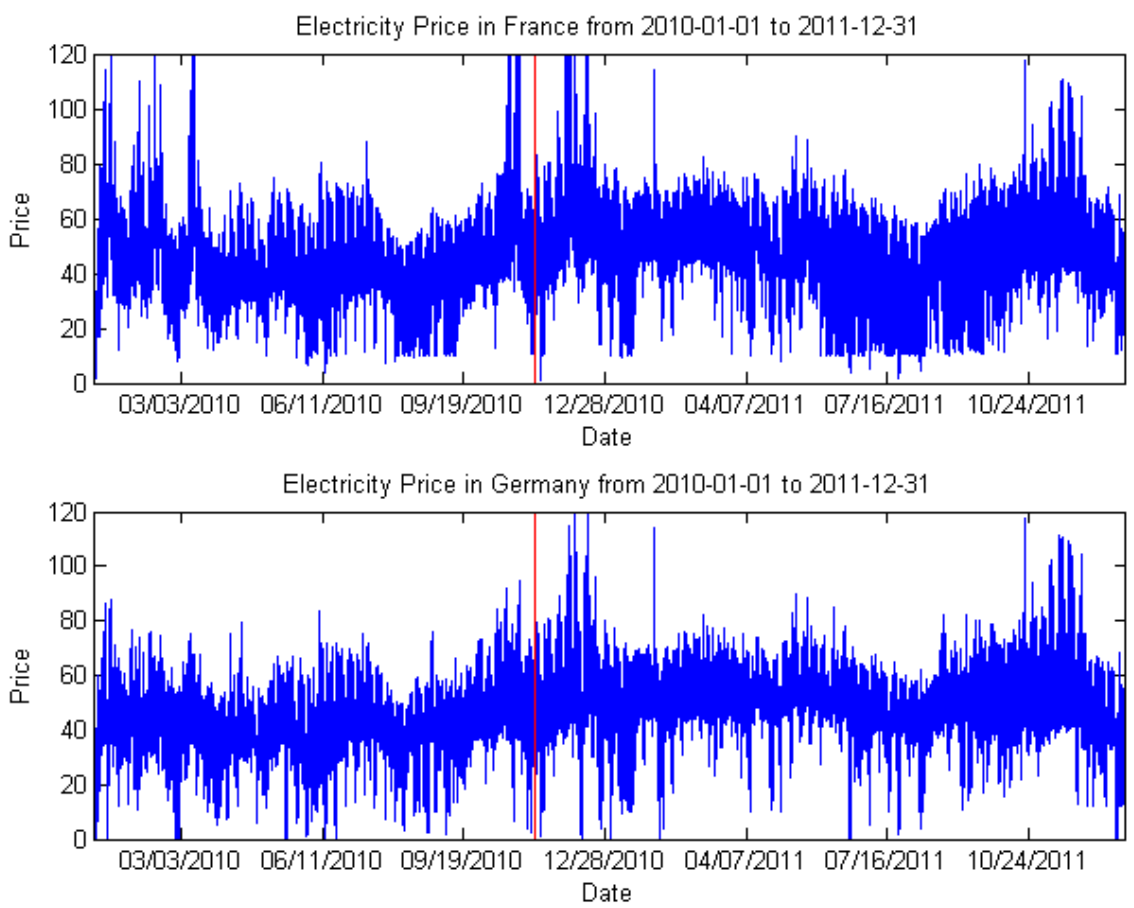

Figure 2: Day-Ahead prices in Germany and France 

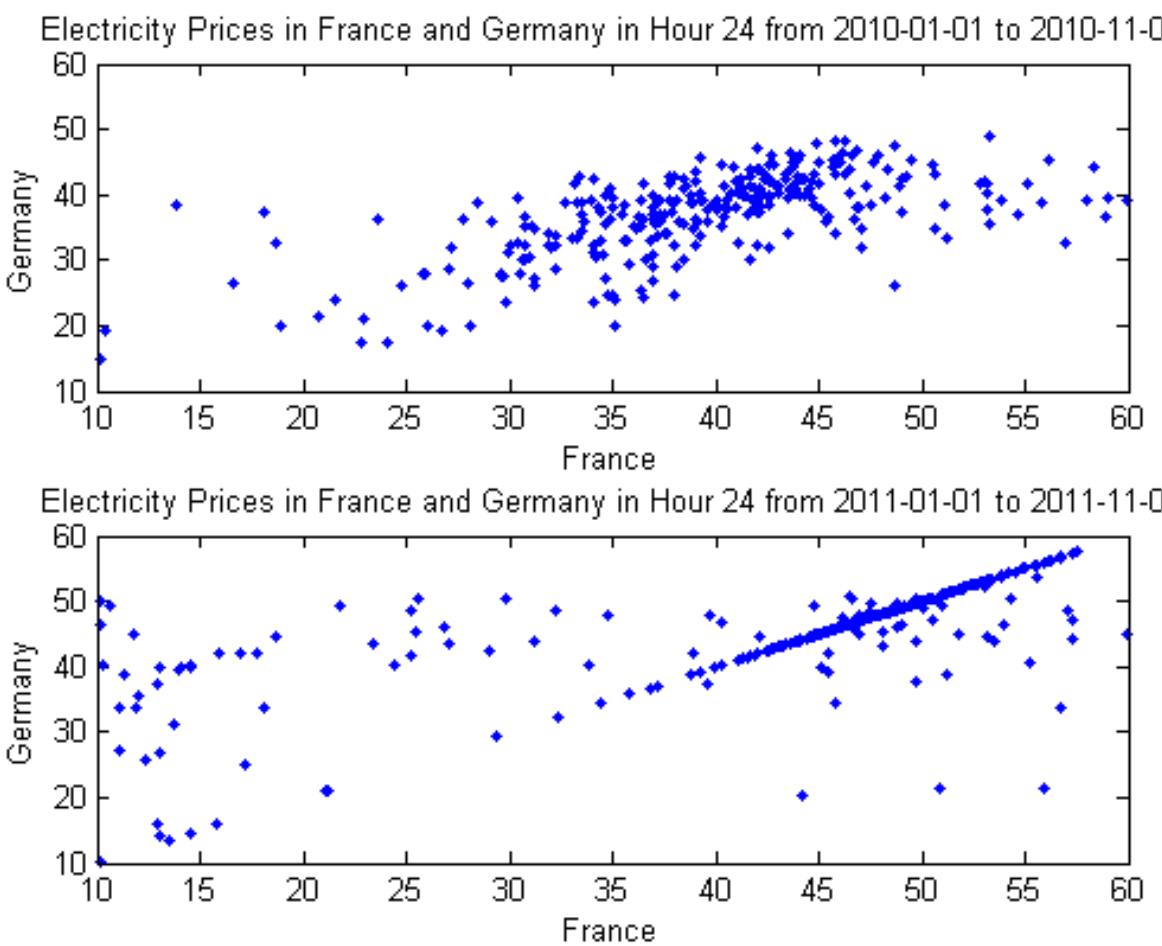

Figure 3: Day-Ahead prices Germany vs France

which induces the demand-filtration $\mathcal{F}^{D}$. The risk factors which drive fuels prices are given by the m-dimensional Brownian Motion

$$
W_{t}^{S}=\left(W_{t}^{S_{1}}, \ldots, W_{t}^{S_{m}}\right) .
$$

$W^{S}$ generates the filtration $\mathcal{F}^{S}$. We assume fuels prices to be unique across markets, i.e. for example gas trades at the same price in each market. As market participants are able to observe load and fuels prices, we define the market filtration as $\mathcal{F}=\mathcal{F}^{D} \vee \mathcal{F}^{S}$.

Price formation in many electricity markets is done by intersecting aggregated demand and supply to find the market clearing price. However, load and demand are not the same. If producers sold electricity in the forward or futures market, they face a make-or-buy desicion in the day-ahead market. This leads to price-sensitive demand in the auction, as producers are willing to buy back electricity and not to produce it in case of low market prices. The actual load however is relatively price-insensitive. In the model, we focus on load instead of demand - although from now on, we use the word demand as synonym for load.

\subsection{Market Supply Curve}

The supply side of the market ist modeled by the so called market supply curve. The market supply curve for country $i$,

$$
C^{i}:\left[0, \bar{\xi}^{i}\right] \times \mathbb{R}_{+}^{m} \rightarrow \mathbb{R}
$$

with $\left(\xi^{i}, S\right) \rightarrow C^{i}\left(\xi^{i}, S\right)$ maps the capacity usage $\xi^{i}$ given fuels prices $S$ to the price of the last bid needed to fulfill the capacity usage. If each generator is fueled by one fuel only, the market 
supply curve in market area $i$ can be decomposed fuel by fuel, leading to the market fuel supply curve

$$
C_{j}^{i}:\left[0, \bar{\xi}_{j}^{i}\right] \times \mathbb{R}_{+} \rightarrow \mathbb{R}
$$

with $\left(\xi_{j}^{i}, S_{j}\right) \rightarrow C_{j}^{i}\left(\xi_{j}^{i}, S_{j}\right)$ mapping the capacity of generators fueld by fuel $j$ used in market area $i$ given a market fuel price of $S_{j}$ for fuel $j$ to the price of the last bid from a fuel- $j$ generator which is needed to fulfill the capacity usage. Analogously to Carmona et al. (2013) we can reconstruct the market supply curve $C^{i}$ given the market fuel supply curves $C_{j}^{i}$ by first defining their generalized (right continous) inverses by

$$
C_{j}^{i}\left(\cdot, S_{j}^{i}\right)^{-1}(p)=\min \left\{\inf \left\{\xi \in\left(0, \bar{\xi}_{j}^{i}\right]: C_{j}^{i}\left(\xi, S_{j}^{i}\right)>p\right\}, \bar{\xi}_{j}^{i}\right\}
$$

with $\inf \emptyset=+\infty$. This gives maps from prices to capacities which are in or at the money at this price. Summing up over all capacities gives the total capacity which is in or at the money. Inverting this function again delivers back the market supply curve:

$$
C^{i}\left(\xi^{i}, S\right)=\inf \left\{p \in \mathbb{R}: \sum_{j \in\{1, \ldots, m\}} C_{j}^{i}\left(\cdot, S_{j}^{i}\right)^{-1}(p)>\xi^{i}\right\} .
$$

However, if there are generators which can be fueled by different fuels, for example a gas fired power plant which can be fired by gasoline as well, the decomposition of the market supply curve by fuel is not anymore possible.

\subsection{Grid Stability and Interconnector Capacity}

As electricity is not storable, the capacity used has to equal demand at all times, leading to

$$
\xi_{t}^{i}=D_{t}^{i} \forall i=1, \ldots, n \forall t \in[0, T]
$$

if markets are not interconnected.

Market areas $i_{1}$ and $i_{2}\left(i_{1}<i_{2}\right)$ are interconnected by a interconnector with capacity $\underline{E}^{i_{1}, i_{2}}$ in the direction from $i_{2}$ to $i_{1}$ and $\bar{E}^{i_{1}, i_{2}}$ in the direction from $i_{1}$ to $i_{2}$. Hence, the interconnector capacity used at time $t, E_{t}^{i_{1}, i_{2}}$, is restricted:

$$
E_{t}^{i_{1}, i_{2}} \in\left[\underline{E}^{i_{1}, i_{2}}, \bar{E}^{i_{1}, i_{2}}\right] \forall t \in[0, T] .
$$

In interconnected markets, the non-storability condition is

$$
\xi_{t}^{i}=D_{t}^{i}-\sum_{i_{1}=1}^{i-1} E_{t}^{i_{1}, i}+\sum_{i_{2}=i+1}^{n} E_{t}^{i, i_{2}}=D_{t}^{i}-\sum_{k=1}^{n} E_{t}^{k, i}=\widehat{D}_{t}^{i} .
$$

where we set $E^{i, i}=0, E^{i_{2}, i_{1}}=-E^{i_{1}, i_{2}} \forall i_{1}<i_{2}$ and $\widehat{D}^{i}$ denotes the residual load after imports and exports in country $i$. Note that as the market supply curve is defined for non-negative capacities only, the non-storability condition implicitely requires $\widehat{D}_{t}^{i} \geq 0$ which is equivalent to $D_{t}^{i} \geq \sum_{k \neq i} E_{t}^{k, i}$, i.e. demand in market area $i$ has to exceed the sum of all imports minus the sum of all exports. 


\subsection{Electricity Prices}

For given vectors $S_{t}$ of fuels prices, $D_{t}$ of electricity demand and a given matrix $E_{t}=\left(E_{t}^{j, i}\right)_{j, i}$ of interconnector usage, the market prices of electricity for all market areas are determined by

$$
\begin{aligned}
P_{t}^{1} & =C^{1}\left(D_{t}^{1}-\sum_{k \neq 1} E^{k, 1}, S_{t}\right) \\
\vdots & \\
P_{t}^{n} & =C^{n}\left(D_{t}^{n}-\sum_{k \neq n} E^{k, n}, S_{t}\right) .
\end{aligned}
$$

I.e. in market area $i$ the residual load after imports and exports $\hat{D}_{t}^{i}=D_{t}^{i}-\sum_{k \neq i} E_{t}^{k, i}$ has to be produced by local production leading to a marginal price of $C^{i}\left(D_{t}^{i}-\sum_{k \neq i} E_{t}^{k, i}, S_{t}\right)$.

\subsection{Market Coupling}

EPEX Spot defines Market Coupling as follows: 'Market coupling uses implicit auctions in which players do not actually receive allocations of cross-border capacity themselves but bid for energy on their exchange. The exchanges then use the available cross-border transmission capacity to minimize the price difference between two or more areas' (see EPEXSpot (2014)). This definition does not specify, which metric $d: \mathbb{R}^{n} \rightarrow \mathbb{R}^{+}$should be used to measure price differences. The generic optimization problem reads as follows

$$
\left\{\begin{array}{l}
d\left(P_{t}^{1}, \ldots, P_{t}^{n}\right) \rightarrow \min \\
\underline{E}^{i_{1}, i_{2}} \leq E_{t}^{i_{1}, i_{2}} \leq \bar{E}^{i_{1}, i_{2}}, \forall i_{1}<i_{2} .
\end{array}\right.
$$

Taking above mentioned definition as basis, the distance measure which minimizes the sum of all price differences between all market areas would be

$$
d(x)=\sum_{i=1}^{n} \sum_{j=1}^{n}\left|x_{i}-x_{j}\right| .
$$

However, as it is stated in Annex B of EPEXSpot (2013), the market coupling algorithm which is really used maximizes social welfare as target function. As we assume price inelastic demand, maximization of social welfare is equivalent to minimizing production costs. Thus, the optimization problem can be written as:

$$
\left\{\begin{array}{l}
\sum_{i=1}^{n} \int_{0}^{D_{t}^{i}-\sum_{k \neq i} E_{t}^{k, i}} C^{i}\left(x_{i}, S_{t}\right) d x_{i} \rightarrow \min \\
\underline{E}^{i_{1}, i_{2}} \leq E_{t}^{i_{1}, i_{2}} \leq \bar{E}^{i_{1}, i_{2}}, \forall i_{1}<i_{2} .
\end{array}\right.
$$

The solution (i.e. the optimal flows) is called $E^{*, i_{1}, i_{2}}, \forall i_{1}<i_{2}$.

\subsubsection{The two market case}

In the special case of only two interconnected markets there is only the interconnector $E^{1,2}$ and the optimization problem becomes one dimensional. In this case, the optimization problems in (14) and (15) are equivalent. 


\section{Proposition 3.1}

The optimization problems

$$
\left\{\begin{array}{l}
\int_{0}^{D_{t}^{1}+E_{t}^{1,2}} C^{1}\left(x_{1}, S_{t}\right) d x_{1}+\int_{0}^{D_{t}^{2}-E_{t}^{1,2}} C^{2}\left(x_{2}, S_{t}\right) d x_{2} \rightarrow \min \\
\underline{E}^{1,2} \leq E_{t}^{1,2} \leq \bar{E}^{1,2}
\end{array}\right.
$$

and

$$
\left\{\begin{array}{l}
\left|C_{t}^{1}\left(D_{1}+E_{t}^{1,2}, S_{t}\right)-C^{2}\left(D_{2}-E_{t}^{1,2}, S_{t}\right)\right| \rightarrow \min \\
\underline{E}^{1,2} \leq E_{t}^{1,2} \leq \bar{E}^{1,2}
\end{array}\right.
$$

yield the same optimal solution $E^{*, 1,2}$.

Proof: Define

$$
h(E)=\int_{0}^{D_{t}^{1}+E} C^{1}\left(x_{1}, S_{t}\right) d x_{1}+\int_{0}^{D_{t}^{2}-E} C^{2}\left(x_{2}, S_{t}\right) d x_{2} .
$$

Then, $h$ is differentiable and $h^{\prime}(E)=C^{1}\left(D_{t}^{1}+E, S_{t}\right)-C^{2}\left(D_{t}^{2}-E, S_{t}\right)$. Moreover, as $C^{i}\left(x, S_{t}\right) \nearrow$ in $x \forall i$ it follows that $h^{\prime}(E) \nearrow$ in $x$.

- Direction $\Rightarrow$ : Assume, $E^{*}$ is optimal for (16). Then, either $h^{\prime}\left(E^{*}\right)=0, h^{\prime}\left(E^{*}\right)>0$ and $E^{*}=\underline{E}^{1,2}$ or $h^{\prime}\left(E^{*}\right)<0$ and $E^{*}=\bar{E}^{1,2}$.

- Case $h^{\prime}\left(E^{*}\right)=0$ : Then, $\left|C_{t}^{1}\left(D_{1}+E^{*}, S_{t}\right)-C^{2}\left(D_{2}-E^{*}, S_{t}\right)\right|=0$ and thus, $E^{*}$ is optimal for (17) as well.

- Case $h^{\prime}\left(E^{*}\right)>0:$ As $h^{\prime} \nearrow$ it follows that $\left|h^{\prime}\right|$ is minimal for $E^{*}=\underline{E}$.

- Case $h^{\prime}\left(E^{*}\right)<0$ : analogous to case $h^{\prime}\left(E^{*}\right)>0$.

- Direction $\Leftarrow$ : Let $E^{*}$ be the solution of (17).

- Case $\left|C_{t}^{1}\left(D_{1}+E^{*}, S_{t}\right)-C^{2}\left(D_{2}-E^{*}, S_{t}\right)\right|=0$ : It follows that $h^{\prime}=0$ and as $h^{\prime} \nearrow$ this implies that $E^{*}$ is optimal for (16).

- Case $C_{t}^{1}\left(D_{1}+E^{*}, S_{t}\right)>C^{2}\left(D_{2}-E^{*}, S_{t}\right)$ : This implies on the one hand $h^{\prime}\left(E^{*}\right)>0$ (by definition of $h$ ) and on the other hand $E^{*}=\underline{E}$ (as $C^{1}\left(x, S_{t}\right) \nearrow$ in $x$ and $C^{2}\left(x, S_{t}\right) \nearrow$ in $x)$. Thus, $h^{\prime}>0$ on the whole intervall. This implies that $h$ attains it's minimum at $\underline{E}$.

- Case $C_{t}^{1}\left(D_{1}+E^{*}, S_{t}\right)<C^{2}\left(D_{2}-E^{*}, S_{t}\right)$ : Analog to the second case.

\subsection{The case of two markets with one fuel and exponential market sup- ply curve}

Explicit calculation of electricity prices according to equations (10) is generally not possible as there is no explicit solution to the optimization problem in equation (15). However, for specific 
forms of the market supply curves, it is possible to calculate closed form solutions for electricity spot prices and (semi-) closed form formulae for their cumulative distribution function. We assume the market supply functions $C^{i}$ to be explicitly given by

$$
C^{i}\left(\xi^{i}, S\right)=S e^{a_{i}+b_{i} \xi^{i}}+c .
$$

The constants $a_{i} \in \mathbb{R}$ and $b_{i}>0$ are given for each market area and all market areas share the same constant $c \in \mathbb{R}$. Note that such a market supply curve is by definition monotonically increasing in capacity used $\left(\xi^{i}\right)$ and fuels prices $(S)$. The electricity prices (conditional on knowledge of interconnector usage) are then given as in equations (10) as

$$
\begin{aligned}
P_{t}^{1}\left(D_{t}^{1}, E_{t}, S_{t}\right) & =S_{t} e^{a_{1}+b_{1}\left(D_{t}^{1}-E_{t}\right)}+c \\
P_{t}^{2}\left(D_{t}^{2},-E_{t}, S_{t}\right) & =S_{t} e^{a_{2}+b_{2}\left(D_{t}^{2}+E_{t}\right)}+c .
\end{aligned}
$$

It is possible to identify three different event sets:

$$
\begin{aligned}
& A_{1}=\left\{\omega \in \Omega: P_{t}^{1}\left(D_{t}^{1}, \bar{E}, S_{t}\right) \geq P_{t}^{2}\left(D_{t}^{2},-\bar{E}, S_{t}\right)\right\} \\
& A_{2}=\left\{\omega \in \Omega: P_{t}^{1}\left(D_{t}^{1}, \underline{E}, S_{t}\right) \leq P_{t}^{2}\left(D_{t}^{2},-\underline{E}, S_{t}\right)\right\} \\
& A_{3}=\Omega \backslash\left(A_{1} \cup A_{2}\right) .
\end{aligned}
$$

$A_{1}$ corresponds to all situations in which the price in market area 1 is higher than the corresponding price in market area 2 although the maximum available capacity of the interconnector is used to transport as much electricity as possible from market area 2 to market area 1. $A_{2}$ corresponds to all situations in which the same is the case in the other direction, i.e. the price in market area 2 is higher than in market area 1 even if the interconnector is used at maximum available capacity to transport electricity from market area 1 to market area 2. Event set $A_{3}$ contains all situations in which price convergence is possible. Market coupling now implies that the optimal interconnector usage is $E^{*}=\bar{E}$ in case of $A_{1}, E^{*}=\underline{E}$ in case of $A_{2}$ and for $A_{3}$ one finds

$$
\begin{array}{r}
P_{t}^{1}\left(D_{t}^{1}, E_{t}^{*}, S_{t}\right) \stackrel{!}{=} P_{t}^{2}\left(D_{t}^{2}, E_{t}^{*}, S_{t}\right) \Rightarrow S_{t} e^{a_{1}+b_{1}\left(D_{t}^{1}-E_{t}^{*}\right)}+c \stackrel{!}{=} S_{t} e^{a_{2}+b_{2}\left(D_{t}^{1}+E_{t}^{*}\right)}+c \Rightarrow \\
a_{1}-a_{2}+b_{1} D_{t}^{1}-b_{2} D_{t}^{2} \stackrel{!}{=}\left(b_{1}+b_{2}\right) E_{t}^{*} \Rightarrow E_{t}^{*}(\omega) \stackrel{!}{=} \frac{a_{1}-a_{2}}{b_{1}+b_{2}}+\frac{b_{1}}{b_{1}+b_{2}} D_{t}^{1}(\omega)-\frac{b_{2}}{b_{1}+b_{2}} D_{t}^{2}(\omega) .
\end{array}
$$

Thus, the optimal interconnector usage is given as

$$
E_{t}^{*}(\omega)= \begin{cases}\bar{E} & \text {,if } \omega \in A_{1} \\ \frac{E}{\frac{a_{1}-a_{2}}{b_{1}+b_{2}}+\frac{b_{1}}{b_{1}+b_{2}} D_{t}^{1}(\omega)-\frac{b_{2}}{b_{1}+b_{2}} D_{t}^{2}(\omega)} & \text {, if } \omega \in A_{2} \\ \text {,if } \omega \in A_{3} .\end{cases}
$$

Let $P_{t}^{*, 1}$ and $P_{t}^{*, 2}$ be the electricity prices at time $t$ in market area 1 and 2 if markets are coupled. I.e. $P_{t}^{*, i}=P_{t}^{i}\left(D_{t}^{i}, E_{t}^{*}, S_{t}\right)$. Then, we find by plugging in (24) into (19)

$$
\begin{array}{r}
P_{t}^{*, 1}(\omega)=P^{1}\left(D_{t}^{1}, E_{t}^{*}, S_{t}\right)= \begin{cases}C^{1}\left(D_{t}^{1}(\omega)-E_{\max }, S_{t}(\omega)\right) & , \text { if } \omega \in A_{1} \\
C^{1}\left(D_{t}^{1}(\omega)-E_{\min }, S_{t}(\omega)\right) & , \text { if } \omega \in A_{2} \\
C^{m}\left(D_{t}^{1}(\omega)+D_{t}^{2}(\omega), S_{t}(\omega)\right) & \text {, if } \omega \in A_{3}\end{cases} \\
P_{t}^{*, 2}(\omega)=P^{2}\left(D_{t}^{2},-E_{t}^{*}, S_{t}\right)= \begin{cases}C^{2}\left(D_{t}^{2}(\omega)+\bar{E}, S_{t}(\omega)\right) & \text {, if } \omega \in A_{1} \\
C^{2}\left(D_{t}^{2}(\omega)+\underline{E}, S_{t}(\omega)\right) & \text {, if } \omega \in A_{2} \\
C^{m}\left(D_{t}^{1}(\omega)+D_{t}^{2}(\omega), S_{t}(\omega)\right) & \text {,if } \omega \in A_{3}\end{cases}
\end{array}
$$

where the function $C^{m}$ can be viewed as the aggregated market supply curve for both countries and is given by

$$
C^{m}(\xi, S)=S e^{a_{m}+b_{m} \xi}+c
$$

with $a_{m}=\frac{a_{1} b_{2}+a_{2} b_{1}}{b_{1}+b_{2}}$ and $b_{m}=\frac{b_{1} b_{2}}{b_{1}+b_{2}}$. 


\subsection{Distributional properties of spot sprices}

Above construction of the spot price model shows how electricity spot prices are related to the demand processes and fuels price. Before introducing specific models for the demand processes and fuels prices let us consider the general case in which the cumulative distribution function of the vector $\left.Z_{t}\right|_{\mathcal{F}_{s}}=\left.\left(D_{t}^{1}, D_{t}^{2}, \ln \left(S_{t}\right)\right)^{T}\right|_{\mathcal{F}_{s}}$ of demand and fuel at time $t$ under the information at time $s(s<t)$ is known. For any matrix $M \in \mathbb{R}^{k, 3}$ we denote by $F_{s, t}^{M}(x)$ the cumulative distribution function of the vector $\left.\left(D_{t}^{1}, D_{t}^{2}, \ln \left(S_{t}\right)\right)^{T}\right|_{\mathcal{F}_{s}}$ premultiplied by $M$ :

$$
\left.M \cdot Z_{t}\right|_{\mathcal{F}_{s}} \sim F_{s, t}^{M} .
$$

The cumulative distribution function of the vector itself is denoted by $F_{s, t}$.

Knowledge of $F_{s, t}^{M}$ allows us to calculate the cumulative distribution function of spot prices by using the partition $\left\{A_{1}, A_{2}, A_{3}\right\}$ of the probability space $\Omega$. Defining $P_{t}^{*}=\left(P_{t}^{*, 1}, P_{t}^{*, 2}\right)^{T}$ the cumulative distribution function $F_{P_{t}^{*} \mid \mathcal{F}_{s}}: \mathbb{R}^{2} \rightarrow \mathbb{R}$ is given by

$$
\begin{aligned}
& F_{\left.P_{t}^{*}\right|_{\mathcal{F}_{s}}}(x)=\mathbb{Q}\left(P_{t}^{*} \leq x \mid \mathcal{F}_{s}\right)= \\
& \mathbb{Q}\left(\left\{P_{t}^{*} \leq x\right\} \cap A_{1} \mid \mathcal{F}_{s}\right)+\mathbb{Q}\left(\left\{P_{t}^{*} \leq x\right\} \cap A_{2} \mid \mathcal{F}_{s}\right)+\mathbb{Q}\left(\left\{P_{t}^{*} \leq x\right\} \cap A_{3} \mid \mathcal{F}_{s}\right)
\end{aligned}
$$

and the probabilities in (29) are calculated as follows:

$$
\begin{aligned}
& \mathbb{Q}\left(\left\{P_{t}^{*} \leq x\right\} \cap A_{1} \mid \mathcal{F}_{s}\right) \\
= & \mathbb{Q}\left(\left\{S_{t} e^{a_{1}+b_{1}\left(D_{t}^{1}-\bar{E}\right)}+c \leq x_{1}\right\} \cap\left\{S_{t} e^{a_{2}+b_{2}\left(D_{t}^{2}+\bar{E}\right)}+c \leq x_{2}\right\} \cap\right. \\
& \left.\left\{S_{t} e^{a_{1}+b_{1}\left(D_{t}^{1}-\bar{E}\right)}+c \geq S_{t} e^{a_{2}+b_{2}\left(D_{t}^{2}+\bar{E}\right)}+c\right\}\right) \\
= & \mathbb{Q}\left(\{ \operatorname { l n } ( S _ { t } ) + b _ { 1 } D _ { t } ^ { 1 } \leq \operatorname { l n } ( x _ { 1 } - c ) - a _ { 1 } + b _ { 1 } \overline { E } \} \cap \left\{\ln \left(S_{t}\right)+b_{2}\left(D_{t}^{2} \leq \ln \left(x_{2}-c\right)-a_{2}-b_{2} \bar{E}\right\} \cap\right.\right. \\
& \left.\left\{-b_{1} D_{t}^{1}+b_{2} D_{t}^{2} \leq a_{1}-a_{2}-\left(b_{1}+b_{2}\right) \bar{E}\right\}\right) \\
= & \mathbb{Q}\left(M_{1} Z_{t} \leq d_{1}(x)\right)=F^{M_{1}}\left(d_{1}(x)\right) .
\end{aligned}
$$

The parameters are

$$
d_{1}(x)=\left(\begin{array}{c}
\ln \left(x_{1}-c\right)-a_{1}+b_{1} \bar{E} \\
\ln \left(x_{2}-c\right)-a_{2}-b_{2} \bar{E} \\
a_{1}-a_{2}-\left(b_{1}+b_{2}\right) \bar{E}
\end{array}\right) \quad, \quad M_{1}=\left(\begin{array}{rrr}
b_{1} & 0 & 1 \\
0 & b_{2} & 1 \\
-b_{1} & b_{2} & 0
\end{array}\right) .
$$

Similar calculations lead to

$$
\mathbb{Q}\left(\left\{P_{t}^{*} \leq x\right\} \cap A_{2} \mid \mathcal{F}_{s}\right)=F^{M_{2}}\left(d_{2}(x)\right)
$$

with

$$
d_{2}(x)=\left(\begin{array}{c}
\ln \left(x_{1}-c\right)-a_{1}+b_{1} \underline{E} \\
\ln \left(x_{2}-c\right)-a_{2}-b_{2} \underline{E} \\
-a_{1}+a_{2}+\left(b_{1}+b_{2}\right) \underline{E}
\end{array}\right) \quad, \quad M_{2}=\left(\begin{array}{rrr}
b_{1} & 0 & 1 \\
0 & b_{2} & 1 \\
b_{1} & -b_{2} & 0
\end{array}\right)
$$

and

$$
\mathbb{Q}\left(\left\{P_{t}^{*} \leq x\right\} \cap A_{3} \mid \mathcal{F}_{s}\right)=F^{M_{3}}\left(d_{3}^{u}(x)\right)-F^{M_{3}}\left(d_{3}^{l}(x)\right)
$$

with

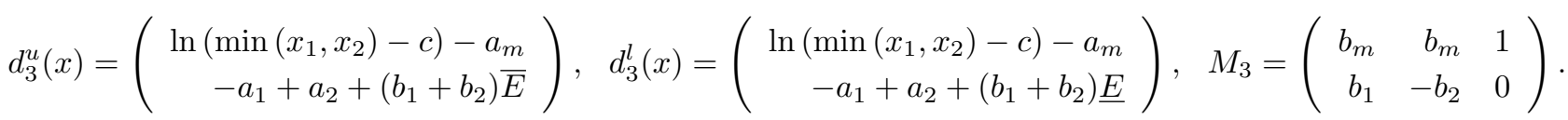

In total, the cumulative distribution function $F_{\left.P_{t}^{*}\right|_{\mathcal{F}_{s}}}(x)$ is given by

$$
F_{\left.P_{t}^{*}\right|_{\mathcal{F}_{s}}}(x)=F^{M_{1}}\left(d_{1}(x)\right)+F^{M_{2}}\left(d_{2}(x)\right)+\left(F^{M_{3}}\left(d_{3}^{u}(x)\right)-F^{M_{3}}\left(d_{3}^{l}(x)\right)\right) .
$$




\subsubsection{Example: Normal demand and lognormal fuels prices}

Under the assumption of a joint multivariate normal distribution of demands and log-fuel prices, i.e.

$$
\left.\left(\begin{array}{l}
D_{t}^{1} \\
D_{t}^{2} \\
\ln \left(S_{t}\right)
\end{array}\right)\right|_{\mathcal{F}_{s}} \sim N(\mu(s, t), \Sigma(s, t))
$$

with parameters $\mu(s, t)$ and $\Sigma(s, t)$ given, the cumulative distribution function $F_{P_{t}^{*} \mid \mathcal{F}_{s}}(x)$ simplifies to

$$
\begin{aligned}
F_{P_{t}^{*} \mid \mathcal{F}_{s}}(x)= & \Phi_{3}\left(d_{1}(x) ; M_{1} \mu(s, t) ; M_{1} \Sigma M_{1}^{T}\right)+ \\
& \Phi_{3}\left(d_{2}(x) ; M_{2} \mu(s, t) ; M_{2} \Sigma M_{2}^{T}\right)+ \\
& \left(\Phi_{2}\left(d_{3}^{u}(x) ; M_{3} \mu(s, t) ; M_{3} \Sigma M_{3}^{T}\right)-\Phi_{2}\left(d_{3}^{l}(x) ; M_{3} \mu(s, t) ; M_{3} \Sigma M_{3}^{T}\right)\right) .
\end{aligned}
$$

As in the extreme case of $\bar{E}=-\underline{E} \rightarrow \infty$ it follows $d_{1}(x) \rightarrow(\infty,-\infty, \infty)^{T}, d_{2}(x) \rightarrow(-\infty, \infty,-\infty)^{T}$, $d_{3}^{u}(x) \rightarrow\left(\ln \left(\min \left(x_{1}, x_{2}\right)-c\right)-a_{m}, \infty\right)^{T}$ and $d_{3}^{l}(x) \rightarrow\left(\ln \left(\min \left(x_{1}, x_{2}\right)-c\right)-a_{m},-\infty\right)^{T}$ we find

$$
F_{P_{t}^{*} \mid \mathcal{F}_{s}}(x) \rightarrow \Phi\left(\ln \left(\min \left(x_{1}, x_{2}\right)-c\right)-a_{m} ; \bar{b}_{m} \mu(s, t) ; \bar{b}_{m}^{T} \Sigma \bar{b}_{m}\right)
$$

with $\bar{b}_{m}=\left(b_{m}, b_{m}, 1\right)^{T}$. This clearly implies identity of electricity prices in both market areas in the almost surely sense.

On the other hand, if $\bar{E}=\underline{E}=0$, it follows that $d_{3}^{u}(x)=d_{3}^{l}(x)$ and

$\Phi_{3}\left(d_{1}(x) ; M_{1} \mu(s, t) ; M_{1} \Sigma M_{1}^{T}\right)+\Phi_{3}\left(d_{2}(x) ; M_{2} \mu(s, t) ; M_{2} \Sigma M_{2}^{T}\right)=\Phi_{2}\left(d_{0}(x) ; M_{0} \mu(s, t) ; M_{0} \Sigma M_{0}\right)$

with $d_{0}(x)=\left(\begin{array}{l}\ln \left(x_{1}-c\right)-a_{1} \\ \ln \left(x_{2}-c\right)-a_{2}\end{array}\right)$ and $M_{0}=\left(\begin{array}{ccc}b_{1} & 0 & 1 \\ 0 & b_{2} & 1\end{array}\right)$. Thus, we find

$$
F_{P_{t}^{*} \mid \mathcal{F}_{s}}(x)=\Phi_{2}\left(d_{0}(x) ; M_{0} \mu(s, t) ; M_{0} \Sigma M_{0}\right) \text {. }
$$

If there is no stochasticity in fuels prices and demand in both market areas are uncorrelated, this implies independence of electricity prices in both market areas. Figure 4 displays a typical scatter plot of electricity prices in one market area versus electricity prices in the other market area. Parameters are choosen such that market area 1 tends to exibit slightly higher prices than market area 2. However, in most of the times, electricity prices coincide in both market areas due to market coupling, i.e. price convergence is attained. This corresponds to event set $A_{3}$. Figure 5 shows the two market areas with the same set of parameters, but instead of typical values for $\bar{E}$ and $\underline{E}$ the extreme cases are plotted. In case of no interconnector capacity, electricity prices exibit correlation due to possible correlation in demand and due to the fact that in both market areas the same fuel is used. If interconnector capacity is available only in one direction, the source market area exhibits at least the electricity price as the sink market area. In case of sufficiently large interconnector capacity, prices in both market areas coincide all the time and thus, prefect price convergence is reached. 


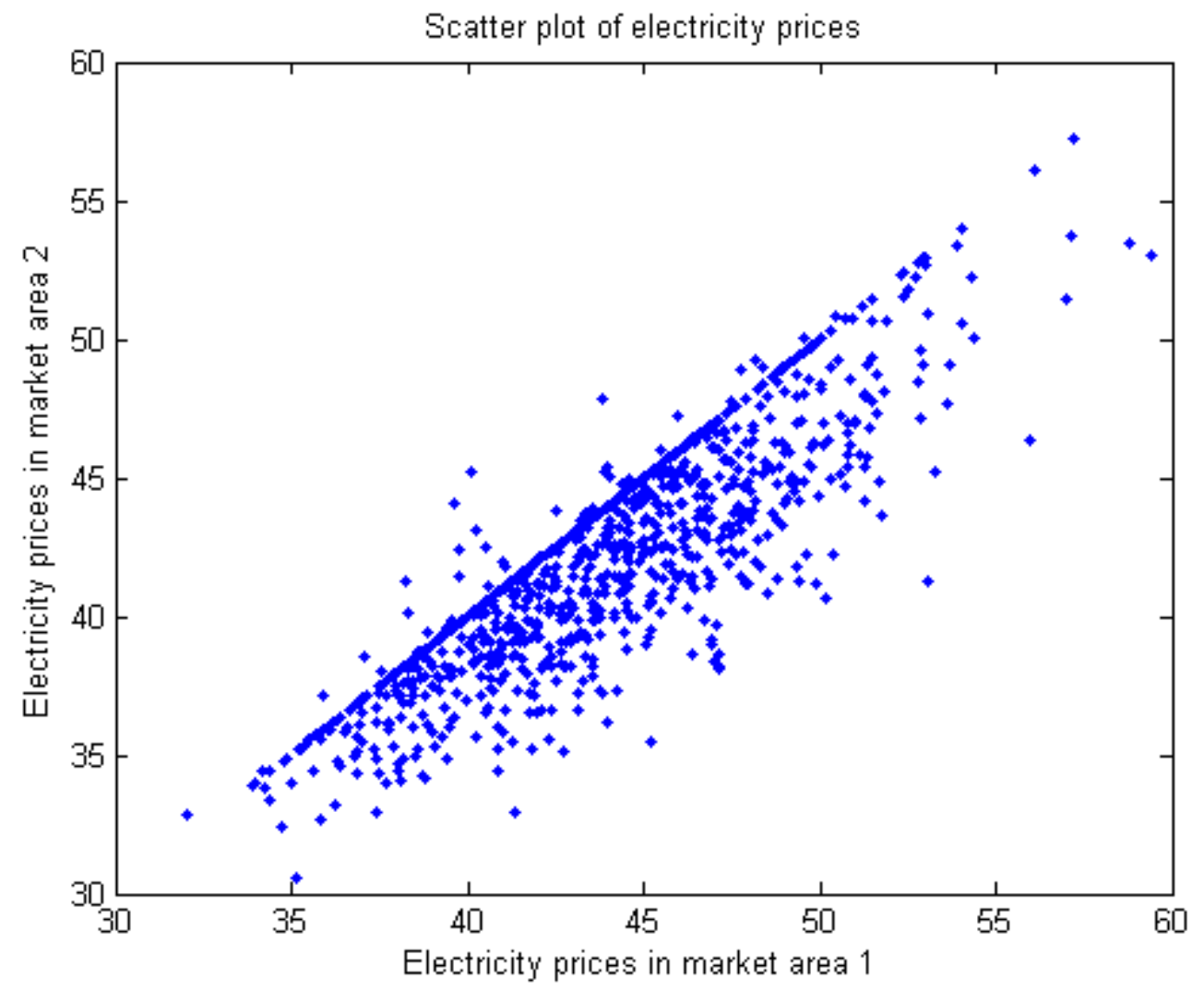

Figure 4: Typical scatter plot of model electricity prices under limited interconnector capacity

\section{HPFCs for Coupled Markets}

As the structural model defines electricity spot prices on a hourly basis, the most basic forward contract which can be analyzed in this model is a hourly forward contract. Fixing a trading date, the function which maps the delivery hour to the forward price for delivery of electricity at this hour is called the hourly price forward curve (HPFC). Standard arbitrage arguments (see Bingham and Kiesel (2004)) imply that the forward price at trading time $s$ for delivery of electricity in market area one at delivery time $t$ which is called $F^{1}(s, t)$ is given as the expected future spot price under the so called pricing meassure $\mathbb{Q}$ :

$$
F^{1}(s, t)=\mathbb{E}_{s}^{\mathbb{Q}}\left[P_{t}^{*, 1}\right] .
$$

We assume that the model is specified under this pricing meassure. This might either be the case as the model is calibrated to market data or (if the model was calibrated under the physical meassure) by assuming that the pricing meassure coincides with the physical meassure for those risk factors which are not traded (demand). Using the partition $\left\{A_{1}, A_{2}, A_{3}\right\}$ it is possible to calculate this expectation:

$$
\begin{aligned}
\mathbb{E}_{s}^{\mathbb{Q}}\left[P_{t}^{*, 1}\right] & =\int_{\Omega} P_{t}^{1}(\omega) \mathbb{Q}(d \omega) \\
& =\int_{A_{1}} C^{1}\left(D_{t}^{1}(\omega)-\bar{E}, S_{t}(\omega)\right) \mathbb{Q}(d \omega) \\
& +\int_{A_{2}} C^{1}\left(D_{t}^{1}(\omega)-\underline{E}, S_{t}(\omega)\right) \mathbb{Q}(d \omega) \\
& +\int_{A_{3}} C^{m}\left(D_{t}^{1}(\omega)+D_{t}^{2}(\omega), S_{t}(\omega)\right) \mathbb{Q}(d \omega)
\end{aligned}
$$

and equivalent for market area 2 .

Generally, there is no closed form solution for these integrals, however, they can be calculated 
No Interconnector Capacity

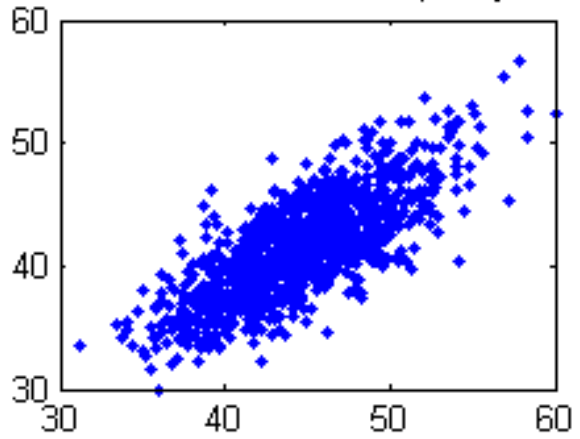

Infinite Capacity from 1 to 2

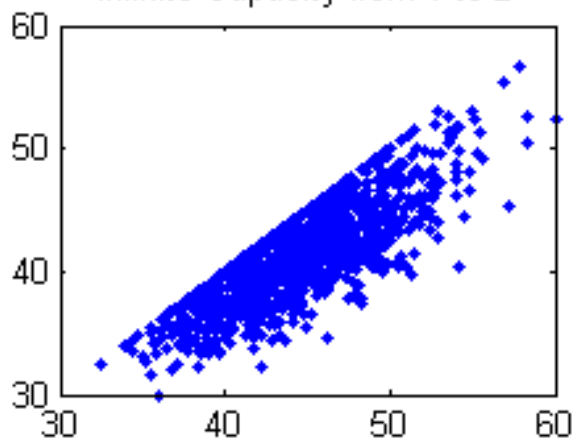

Infinite Capacity from 2 to 1

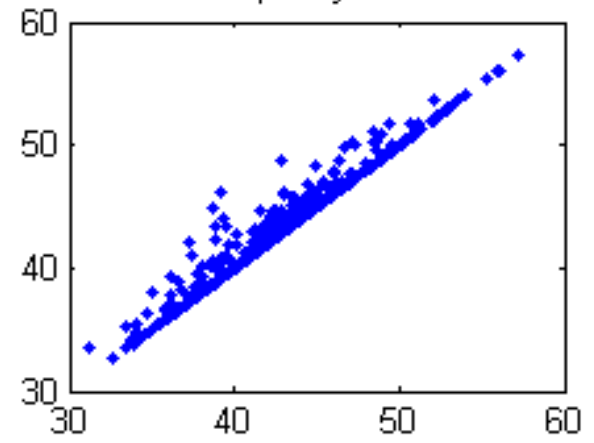

Infinite Capacity

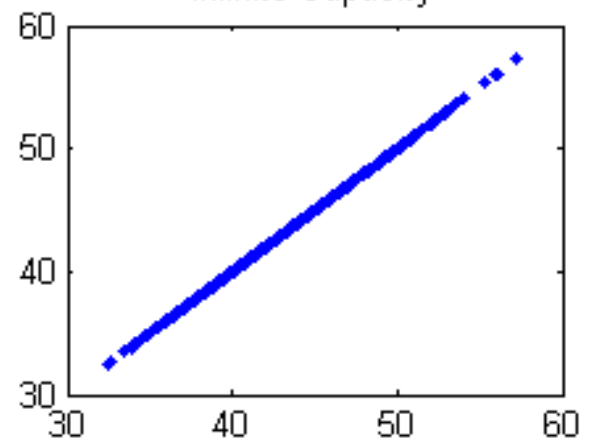

Figure 5: Scatter plots of model electricity prices under varying interconnector capacities

numerically by means of the cumulative distribution function $F_{s, t}(x)$ :

$$
\begin{array}{ll}
\int_{A_{1}} C^{1}\left(D_{t}^{1}(\omega)-\bar{E}, S_{t}(\omega)\right) \mathbb{Q}(d \omega) & =\int_{K_{1}}\left(e^{x_{3}+a_{1}+b_{1}\left(x_{1}-\bar{E}\right)}+c\right) F_{s, t}(d x) \\
\int_{A_{2}} C^{1}\left(D_{t}^{1}(\omega)-\underline{E}, S_{t}(\omega)\right) \mathbb{Q}(d \omega) & =\int_{K_{2}}\left(e^{x_{3}+a_{1}+b_{1}\left(x_{1}-\underline{E}\right)}+c\right) F_{s, t}(d x) \\
\int_{A_{3}} C^{m}\left(D_{t}^{1}(\omega)+D_{t}^{2}(\omega), S_{t}(\omega)\right) \mathbb{Q}(d \omega) & =\int_{K_{3}}\left(e^{x_{3}+a_{m}+b_{m}\left(x_{1}+x_{2}\right)}+c\right) F_{s, t}(d x) .
\end{array}
$$

Here, the areas of integration are

$$
\begin{aligned}
& K_{1}=\left\{x \in \mathbb{R}^{3}: a_{1}+b_{1}\left(x_{1}-\bar{E}\right) \geq a_{2}+b_{2}\left(x_{2}+\bar{E}\right)\right\} \\
& K_{2}=\left\{x \in \mathbb{R}^{3}: a_{1}+b_{1}\left(x_{1}-\underline{E}\right) \leq a_{2}+b_{2}\left(x_{2}+\underline{E}\right)\right\} \\
& K_{3}=\left\{x \in \mathbb{R}^{3}: a_{2}+b_{2}\left(x_{2}+\underline{E}\right)+b_{1} \underline{E} \leq a_{1}+b_{1} x_{1} \leq a_{2}+b_{2}\left(x_{2}+\bar{E}\right)+b_{1} \bar{E}\right\} .
\end{aligned}
$$

However, traded futures are most commonly contracts with a delivery period which exceeds one hour. Thus, to price such a contract let $\mathbb{T}$ denote the set of all hours, in which the contract guarantees the delivery of $1 \mathrm{MW}$. Ignoring interest rate effects (i.e. assuming interest rates to be zero) the price per MWh of such a traded future with delivery in all hours $\mathbb{T}$ can be calculated as

$$
F^{i}(s, \mathbb{T})=\frac{1}{|\mathbb{T}|} \sum_{t \in \mathbb{T}} F^{i}(s, t), \forall i=1,2 .
$$




\subsubsection{Example: Normal demand and lognormal fuels prices}

Under the assumption of a joint multivariate normal distribution of demands and log-fuel prices as in the previous section (see equation (32)), i.e.

$$
\left.\left(\begin{array}{l}
D_{t}^{1} \\
D_{t}^{2} \\
\ln \left(S_{t}\right)
\end{array}\right)\right|_{\mathcal{F}_{s}} \sim N(\mu(s, t), \Sigma(s, t))
$$

with parameters $\mu(s, t)$ and $\Sigma(s, t)$ given, forward prices can be calculated explicitely. Indeed, for the first integral in (37) we find:

$$
\begin{aligned}
\int_{A_{1}} C^{1}\left(D_{t}^{1}(\omega)-\bar{E}, S_{t}(\omega)\right) \mathbb{Q}(d \omega) & =\int_{A_{1}}\left(e^{\ln \left(S_{t}\right)+a_{1}+b_{1}\left(D_{t}^{1}-\bar{E}\right)}+c\right) d \mathbb{Q} \\
& =e^{a_{1}-b_{1} \bar{E}} \int_{A_{1}}\left(e^{\left.\ln \left(S_{t}\right)+b_{1} D_{t}^{1}\right)} d \mathbb{Q}+c \mathbb{Q}\left(A_{1}\right)\right. \\
& =e^{\left(a_{1}-b_{1} \bar{E}+\bar{b}_{3}^{T} \mu(s, t)+\frac{1}{2} \bar{b}_{3}^{T} \Sigma(s, t) \bar{b}_{3}\right)} \Phi\left(\frac{a_{1}-a_{2}-\left(b_{1}+b_{2}\right) \bar{E}-\bar{b}_{3}^{T} \mu(s, t)-\bar{b}_{3}^{T} \Sigma(s, t) \bar{b}_{3}}{\sqrt{\bar{b}_{3}^{T} \Sigma(s, t) \bar{b}_{3}}}\right) \\
& +c \Phi\left(\frac{a_{1}-a_{2}-\left(b_{1}+b_{2}\right) \bar{E}-\bar{b}_{3}^{T} \mu(s, t)}{\sqrt{\bar{b}_{3}^{T} \Sigma(s, t) \bar{b}_{3}}}\right)
\end{aligned}
$$

with $\bar{b}_{3}^{T}=\left(-b_{1}, b_{2}, 0\right)$. Here, we used for the last equality the fact that for normal densities $f_{(\mu, \Sigma)}$ with mean $\mu$ and variance $\Sigma$ it holds for any vector $c$ that

$$
e^{c^{T} z} f_{(\mu, \Sigma)}(z)=e^{\mu^{T} c+c^{T} \Sigma c} f_{(\mu+\Sigma c, \Sigma)}(z), \forall z \in \mathbb{R}^{n}
$$

Figure 6 plots futures prices for both countries for delivery of electricity in one specific hour in the future assuming diffenrent levels of interconnector capacity. There are two situations plotted: The situation corresponding to the dashed lines indicates the behaviour of futures prices if demand in both countries is highly correlated (correlation coefficient of 0.8 ). The situation corresponding to the solid lines indicates the behaviour of futures prices if demand in both countries is uncorrelated. It needs to be stressed that correlation refers to the stochastic part of the demand process. If the periodic pattern in both countries is similar, of course the time series of demand would indicate high correlations. However, after de-periodisation, this might not be true anymore. In this figure, the futures price in country 1 is significantly lower than in country 2 if no interconnector capacity exists. Examining the situation of high demand correlation, futures prices in country 1 are higher, the higer interconnector capacity is. On the other hand, futures prices in country 2 decrease with increasing interconnector capacity. This finally leads to price convergence of both markets. This behavior is exactly as one would intuitively expect. With increasing interconnector capacity, more electricity is transferred form the cheap country to the more expensive country at delivery. This leads to lower prices in the more expensive country and higher demand (due to exports) and thus higher prices (as the supply curve is strictly increasing) in the exporting country. As futures prices are expected future spot prices, this leads to higher futures prices in the exporting country and lower futures prices in the importing country. However, the picture changes as one looks at the situation of uncorrelated demand. Here, futures prices in the more expensive country react just as expected. They decrease with increasing installed interconnector capacity. Futures prices in the cheaper country however decrease as well with increasing interconnector capacity - as long as the capacity is below a certain threshold. This behavior is not intuitive, expected future spot prices decrease if the interconnector capacity - and thus the ability to export to the more expensive country (in most of the $\omega \in \Omega)$ increases. This behavior can be explained as follows: Although spot prices in 


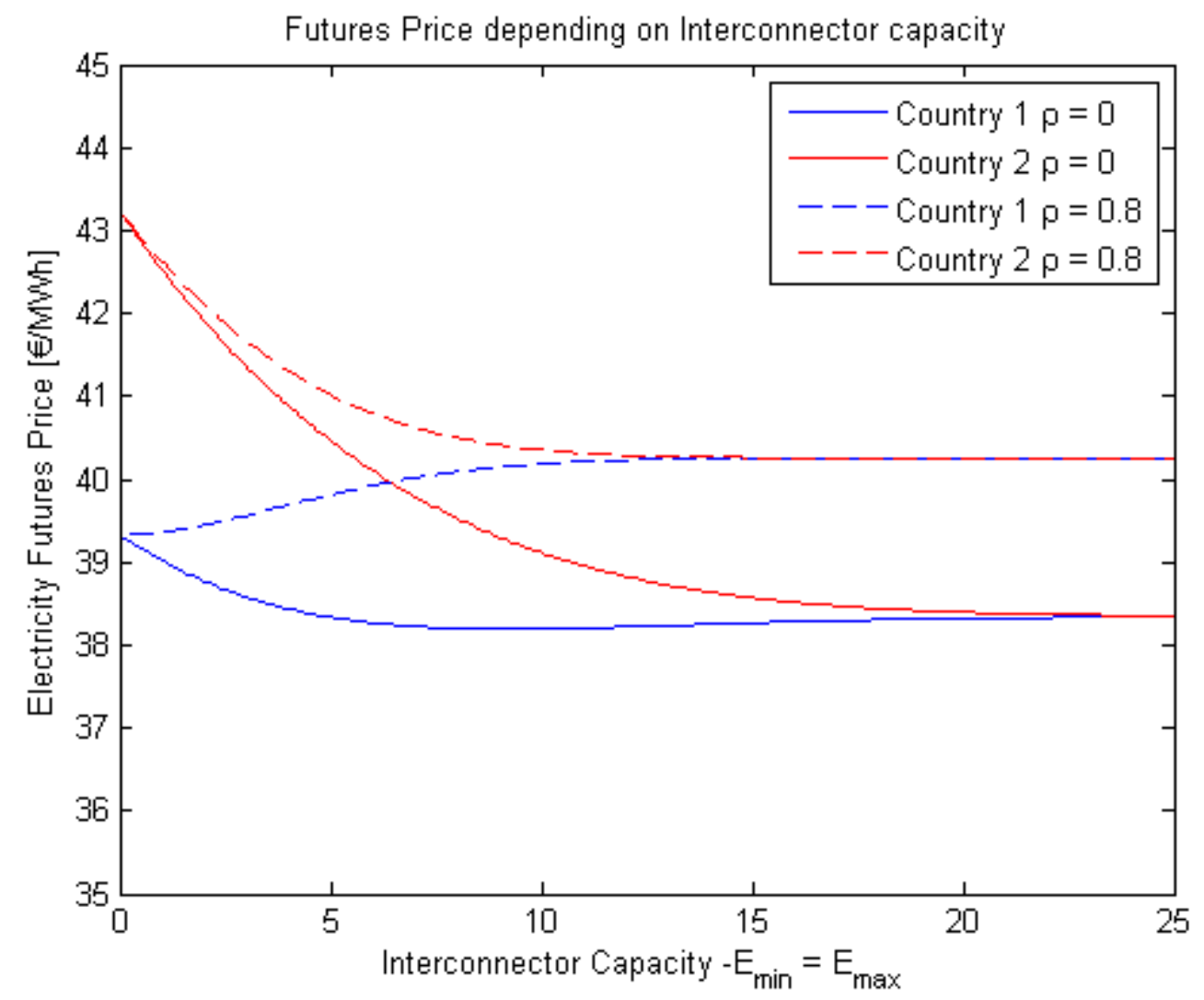

Figure 6: Model futures prices for both countries depending on installed interconnector capacity and demand correlation 
country 1 are cheaper for most of the $\omega \in \Omega$, there is a subset $\Omega_{1} \subset \Omega$ for which electricity prices in country 1 spike whereas country 2 shows relatively low spot prices. If there is interconnector capacity available, on this subset the electricity flow is from country 2 to country 1 and thus decreasing the spot price in country 1 . If $\mathbb{Q}\left(\Omega_{1}\right)$ is big enough (i.e. if correlation is low enough), this effect which lowers expected spot prices in country 1 superimposes the effect of exporting from country 1 on $\Omega \backslash \Omega_{1}$. This line of reasoning is along the lines of a sort of spike-shaving argument for interconnector capacities. As an interconnector reduces the possibility of extreme (upward) spikes, futures prices are lower in the presence of an interconnector as market participants do not have to factor in very high spot prices when pricing futures. Viewing this behavior from a slightly different perspective, one could argue in the following way: Looking at residual demand - which is local demand minus imports plus exports - the presence of an interconnector might increase residual demand in country 1 on average and thus, due to a monotonically increasing market supply curve, lead to increased futures prices. But at the same time, variability of residual demand is reduced. Reduced variability in residual demand combined with a convex market supply curve leads due to Jensen's inequality to lower expected future spot prices. These two effects influence the futures price and depending on which is stronger, might lead to lower futures prices even in country 1.

\section{Option Pricing}

This section deals with the pricing of options in the structural setting for coupled markets presented above. The simplest type of option which can be priced is the european type plain vanilla option written on the hourly electricity price in one of the countries with a certain maturity $t$. Its payoff is simply given as

$$
\left(P_{t}^{*, 1}-K\right)^{+}
$$

in case the option is written on electricity in country 1 and has a strike of $K$. Ignoring interest rates (which would lead to simple discounting only) the value at time $s, V_{s}$, of such an option should be given (see Bingham and Kiesel (2004)) as

$$
V_{s}=\mathbb{E}_{s}^{\mathbb{Q}}\left[\left(P_{t}^{*, 1}-K\right)^{+}\right]
$$

Clearly, this option is a derivative written on fuel, demand in country 1 and demand in country 2. Valuing a plain vanilla option is done by applying the same techniques as for valuing futures. Define three different event sets

$$
\begin{aligned}
& B_{1}=\left\{\omega \in \Omega: C^{1}\left(D_{t}^{1}-\bar{E}, S_{t}\right) \geq K\right\} \\
& B_{2}=\left\{\omega \in \Omega: C^{1}\left(D_{t}^{1}-\underline{E}, S_{t}\right) \geq K\right\} \\
& B_{3}=\left\{\omega \in \Omega: C^{m}\left(D_{t}^{1}+D_{t}^{2}, S_{t}\right) \geq K\right\} .
\end{aligned}
$$

They are closely related to the partition $\left\{A_{1}, A_{2}, A_{3}\right\}$ in the following way: $B_{1}$ contains all situations in which the electricity price in country 1 is higher than $K$ even if the maximum possible amount of electricity $(\bar{E})$ is importet into country $1 . B_{2}$ is the set containing all situations in which the electricity price in country 1 is higher than $K$ even if the maxumum possible amount of electricity $(\underline{E})$ is exportet from country 1 . It clearly holds that $B_{1} \subset B_{2} \cdot B_{3}$ contains all situations in which the combined market of country 1 and country 2 (i.e. the market which would materialize if no interconnector constraints were present) would exhibit an electricity price of $K$ or higher. Note that $B_{3}$ is not a subset of $B_{2}$ nor a superset of $B_{1}$. It holds:

$$
\left\{\omega \in \Omega: P_{t}^{*, 1} \geq K\right\}=\left(A_{1} \cap B_{1}\right) \cup\left(A_{2} \cap B_{2}\right) \cup\left(A_{3} \cap B_{3}\right) .
$$


Having this equality at hand, equation (43) can be calculated as follows:

$$
\begin{aligned}
& \mathbb{E}_{s}^{\mathbb{Q}}\left[\left(P_{t}^{*, 1}-K\right)^{+}\right]=\int_{\left\{\omega \in \Omega: P_{t}^{*, 1} \geq K\right\}} P_{t}^{*, 1}(\omega) d \mathbb{Q}(\omega)-K \mathbb{Q}\left(\left\{\omega \in \Omega: P_{t}^{*, 1} \geq K\right\}\right) \\
& =\int_{A_{1} \cap B_{1}} C^{1}\left(D_{t}^{1}-\bar{E}, S_{t}\right) d \mathbb{Q} \\
& +\int_{A_{2} \cap B_{2}} C^{1}\left(D_{t}^{1}-\underline{E}, S_{t}\right) d \mathbb{Q} \\
& +\int_{A_{3} \cap B_{3}} C^{m}\left(D_{t}^{1}+D_{t}^{2}, S_{t}\right) d \mathbb{Q} \\
& -K\left(\mathbb{Q}\left(A_{1} \cap B_{1}\right)+\mathbb{Q}\left(A_{2} \cap B_{2}\right)+\mathbb{Q}\left(A_{3} \cap B_{3}\right)\right) \\
& =e^{a_{1}-b_{1} \bar{E}} \int_{A_{1} \cap B_{1}} e^{\ln \left(S_{t}\right)+b_{1} D_{t}^{1}} d \mathbb{Q} \\
& +e^{a_{1}-b_{1} \underline{E}} \int_{A_{2} \cap B_{2}} e^{\ln \left(S_{t}\right)+b_{1} D_{t}^{1}} d \mathbb{Q} \\
& +e^{a_{m}} \int_{A_{3} \cap B_{3}} e^{\ln \left(S_{t}\right)+b_{m}\left(D_{t}^{1}+D_{t}^{2}\right)} d \mathbb{Q} \\
& -\tilde{K}\left(\mathbb{Q}\left(A_{1} \cap B_{1}\right)+\mathbb{Q}\left(A_{2} \cap B_{2}\right)+\mathbb{Q}\left(A_{3} \cap B_{3}\right)\right)
\end{aligned}
$$

Here, $\tilde{K}=K-c$ is the adjusted strike price. Figure 7 schematically indicates the form and shapes of the event sets as functions of demands. For this figure, fuel price is hold constant. If the
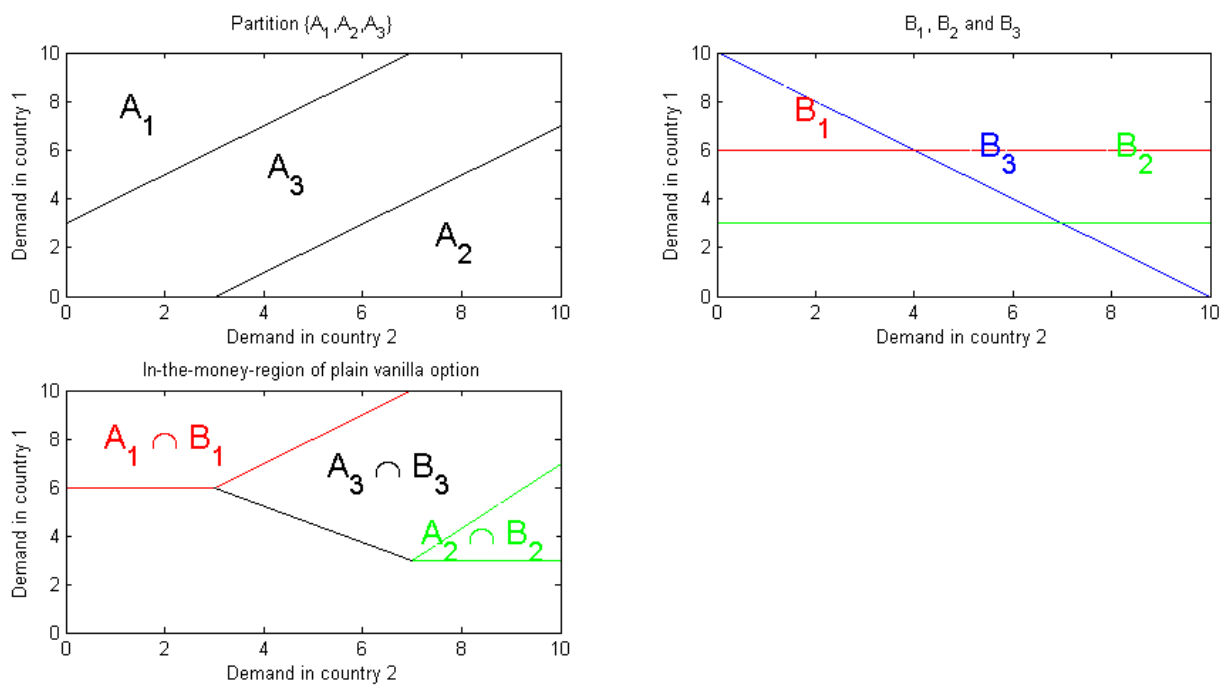

Figure 7: In-the-money-regions for plain vanilla option on spot price in country 1

joint cumulative distribution function of demands and fuel price is known, these integrals can be calculated in terms of numerical quadrature by first rewriting the areas of integration:

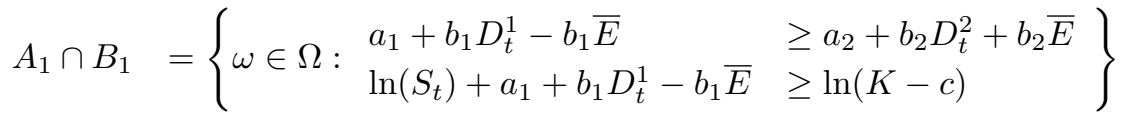

$$
\begin{aligned}
& =\left\{\omega \in \Omega:\left.M_{4} Z_{t}\right|_{\mathcal{F}_{s}} \leq d_{4}\right\}
\end{aligned}
$$

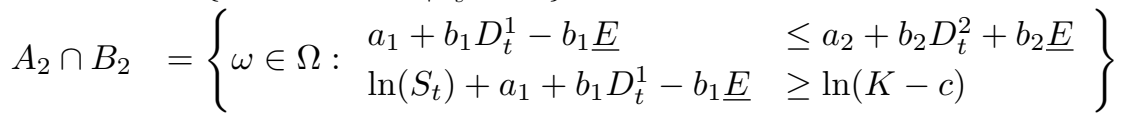

$$
\begin{aligned}
& =\left\{\omega \in \Omega:\left.M_{5} Z_{t}\right|_{\mathcal{F}_{s}} \leq d_{5}\right\}
\end{aligned}
$$

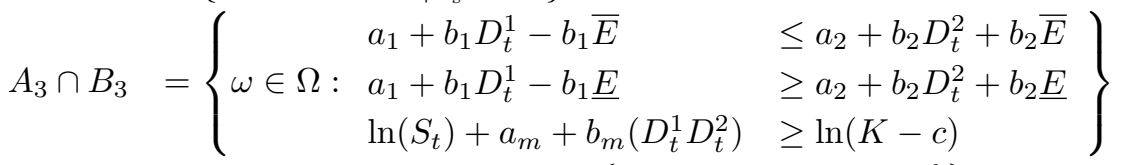

$$
\begin{aligned}
& =\left\{\omega \in \Omega:\left.M_{6} Z_{t}\right|_{\mathcal{F}_{s}} \leq d_{6}^{u}\right\} \backslash\left\{\omega \in \Omega:\left.M_{6} Z_{t}\right|_{\mathcal{F}_{s}} \leq d_{6}^{l}\right\} \text {. }
\end{aligned}
$$


The parameters are

$$
\begin{aligned}
& M_{4}=\left(\begin{array}{lll}
-b_{1} & b_{2} & 0 \\
-b_{1} & 0 & -1
\end{array}\right) d_{4}=\left(\begin{array}{l}
a_{1}-a_{2}-\left(b_{1}+b_{2}\right) \bar{E} \\
a_{1}-b_{1} \bar{E}-\ln (K-c)
\end{array}\right) \\
& M_{5}=\left(\begin{array}{lll}
b_{1} & -b_{2} & 0 \\
-b_{1} & 0 & -1
\end{array}\right) d_{5}=\left(\begin{array}{l}
-a_{1}+a_{2}-\left(b_{1}+b_{2}\right) \underline{E} \\
a_{1}-b_{1} \underline{E}-\ln (K-c)
\end{array}\right) \\
& M_{6}=\left(\begin{array}{lll}
b_{1} & -b_{2} & 0 \\
-b_{m} & -b_{m} & -1
\end{array}\right) \quad d_{6}^{u}=\left(\begin{array}{l}
u \\
a_{m}-\ln (K-c)
\end{array}\right) \quad d_{6}^{l}=\left(\begin{array}{l}
-b_{1}+a_{2}+\left(b_{1}+b_{2}\right) \underline{E} \\
a_{m}-\ln (K-c)
\end{array}\right) .
\end{aligned}
$$

Now, the integrals can be stated with respect to the cumulative distribution function:

$$
\int_{A_{1} \cap B_{1}} e^{\ln \left(S_{t}\right)+b_{1} D_{t}^{1}} d \mathbb{Q}=\int_{K_{4}}\left(e^{x_{3}+b_{1} x_{1}}\right) d F_{s, t}
$$

with $K_{4}=\left\{x \in R^{3}: M_{4} x \leq d_{4}\right\}$ and similar for the other integrals.

\subsubsection{Example: Normal demand and lognormal fuels prices}

The multidimensional numerical quadrature might become quite complicated and unstable, however, for special distributions there are semi-closed form formulae available. Assuming again a joint multivariate normal distribution of demands and log-fuel prices as in equation (32), i.e.

$$
\left.Z_{t}\right|_{\mathcal{F}_{s}}=\left.\left(\begin{array}{l}
D_{t}^{1} \\
D_{t}^{2} \\
\ln \left(S_{t}\right)
\end{array}\right)\right|_{\mathcal{F}_{s}} \sim N(\mu(s, t), \Sigma(s, t))
$$

it follows:

$$
\begin{aligned}
& \mathbb{Q}\left(A_{1} \cap B_{1}\right)=\Phi_{2}\left(d_{4}, M_{4} \mu(s, t), M_{4} \Sigma(s, t) M_{4}^{T}\right) \\
& \mathbb{Q}\left(A_{2} \cap B_{2}\right)=\Phi_{2}\left(d_{5}, M_{5} \mu(s, t), M_{5} \Sigma(s, t) M_{5}^{T}\right) \\
& \mathbb{Q}\left(A_{3} \cap B_{3}\right)=\Phi_{2}\left(d_{6}^{u}, M_{6} \mu(s, t), M_{6} \Sigma(s, t) M_{6}^{T}\right)-\Phi_{2}\left(d_{6}^{l}, M_{6} \mu(s, t), M_{6} \Sigma(s, t) M_{6}^{T}\right)
\end{aligned}
$$

and

$$
\begin{aligned}
\int_{A_{1} \cap B_{1}} e^{\ln \left(S_{t}\right)+b_{1} D_{t}^{1}} d \mathbb{Q} & =\int_{K_{4}}\left(e^{x_{3}+b_{1} x_{1}}\right) f_{(\mu, \Sigma)}(x) d x \\
& =e^{\mu^{T} \bar{b}_{1}+\frac{1}{2} \bar{b}_{1}^{T} \Sigma \bar{b}_{1}} \int_{K_{4}} f_{\left(\mu+\Sigma \bar{b}_{1}, \Sigma\right)}(x) d x \\
& =e^{\mu^{T} \bar{b}_{1}+\frac{1}{2} \bar{b}_{1}^{T} \Sigma \bar{b}_{1}} \Phi_{2}\left(d_{4}, M_{4}\left(\mu(s, t)+\Sigma(s, t) \bar{b}_{1}\right), M_{4} \Sigma(s, t) M_{4}^{T}\right)
\end{aligned}
$$

with $\bar{b}_{1}=\left(b_{1}, 0,1\right)^{T}$ and using the same argument as in equation (41). The same calculations can be done for the other two cases leading to the option price formula

$$
\begin{aligned}
V_{s} & =e^{a_{1}-b_{1} \bar{E}+\mu(s, t)^{T} \bar{b}_{1}+\frac{1}{2} \bar{b}_{1}^{T} \Sigma(s, t) \bar{b}_{1}} \Phi_{2}\left(d_{4}, M_{4}\left(\mu(s, t)+\Sigma(s, t) \bar{b}_{1}\right), M_{4} \Sigma(s, t) M_{4}^{T}\right) \\
& +e^{a_{1}-b_{1} \underline{E}+\mu(s, t)^{T} \bar{b}_{1}+\frac{1}{2} \bar{b}_{1}^{T} \Sigma(s, t) \bar{b}_{1}} \Phi_{2}\left(d_{5}, M_{5}\left(\mu(s, t)+\Sigma(s, t) \bar{b}_{1}\right), M_{5} \Sigma(s, t) M_{5}^{T}\right) \\
& +e^{a_{m}+\mu(s, t)^{T} \bar{b}_{m}+\frac{1}{2} \bar{b}_{m}^{T} \Sigma(s, t) \bar{b}_{m}}\left(\Phi_{2}\left(d_{6}^{u}, M_{6}\left(\mu(s, t)+\Sigma(s, t) \bar{b}_{m}\right), M_{6} \Sigma(s, t) M_{6}^{T}\right)\right. \\
& \left.-\Phi_{2}\left(d_{6}^{l}, M_{6}\left(\mu(s, t)+\Sigma(s, t) \bar{b}_{m}\right), M_{6} \Sigma(s, t) M_{6}^{T}\right)\right) \\
& -\tilde{K}\left(\Phi_{2}\left(d_{4}, M_{4} \mu(s, t), M_{4} \Sigma(s, t) M_{4}^{T}\right)+\Phi_{2}\left(d_{5}, M_{5} \mu(s, t), M_{5} \Sigma(s, t) M_{5}^{T}\right)\right. \\
& \left.+\Phi_{2}\left(d_{6}^{u}, M_{6} \mu(s, t), M_{6} \Sigma(s, t) M_{6}^{T}\right)-\Phi_{2}\left(d_{6}^{l}, M_{6} \mu(s, t), M_{6} \Sigma(s, t) M_{6}^{T}\right)\right)
\end{aligned}
$$

with $\bar{b}_{m}=\left(b_{m}, b_{m}, 1\right)^{T}$.

Figure 8 plots option prices and implied volatilities for both countries using the same parameters as in the previous section. Demand correlation is assumed to be zero and interconnector capacities 

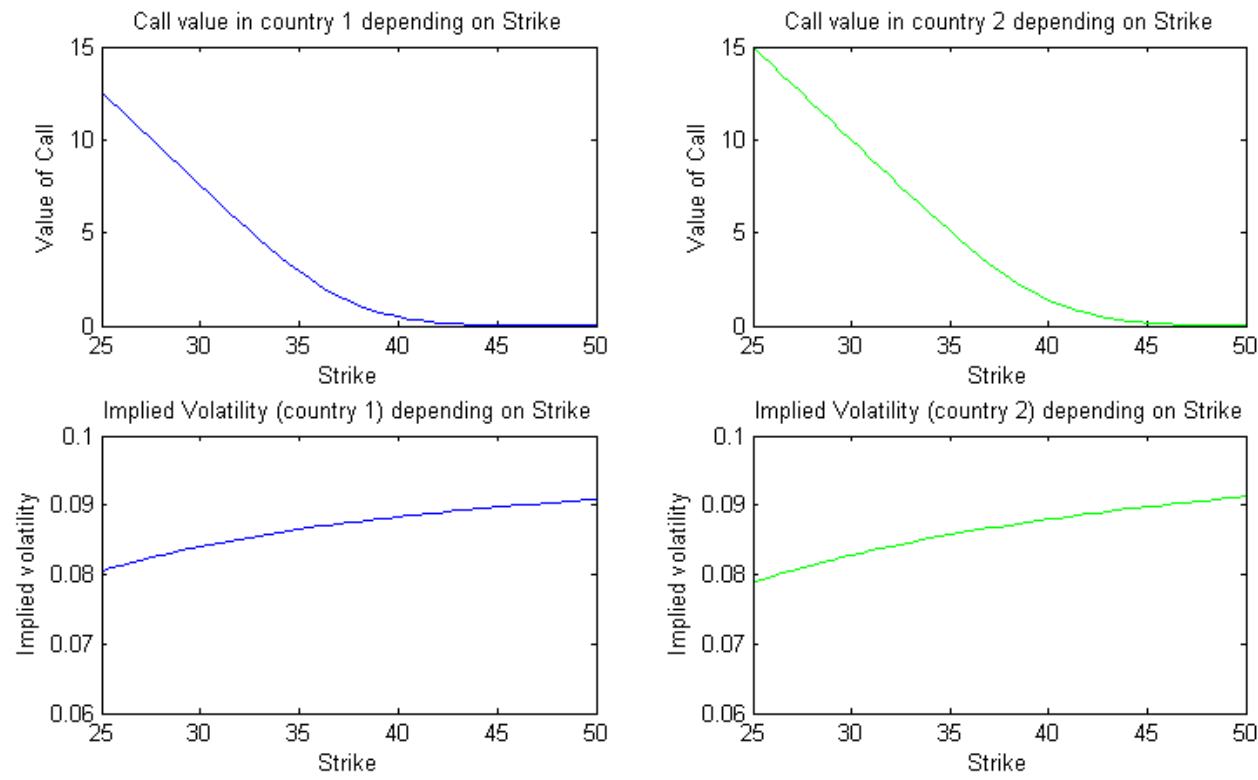

Figure 8: Model option prices for both countries depending on Strikes

are assumed to be 3 in both directions. Option prices exhibit all features which are expected: convex in strikes, asymptotically (i.e. for very little and very big strikes) no positive time-value, time value attains its maximum for at the money options. Backing out the black-implied volatilities of these options, the so called inverse leverage effect becomes visible. Electricity options with higher Strikes are priced with higher implied volatilities. This is not a specific property of interconnected electricity markets but rather due to the convexity of the market supply curve. Knittel and Roberts (2005) are one of the first to report on this inverse leverage effect for deregulated wholesale electricity markets in an empirical study. Figure 9 plots again futures prices for both countries depending on interconnector capacity as in figure 6 and additionally the implied volatility of options written at-the-money, i.e. with Strike equal to the current futures price. In country 1, the futures price increases with increasing interconnector capacity but the implied volatility increases only up to a certain maximum and drops afterwards to levels lower than before. For country 2, the futures prices converge downwards and at the same time the implied volatility decreases as well. Finally, both, the futures prices and the implied at-the-money volatilities converge to common levels. The structure of the implied volatility in country 2 is as expected, the more interconnected the markets are, the lesser the variability of the residual demand after im- and exports and thus, the lower the implied volatility. On the other hand, in country 1 the implied volatility of separated markets is lower than in country 2. Interconnecting the markets leads to a spill-over effect of volatility into country 1 which increases the implied volatility for positive but low levels of interconnector capacity. For higher levels of interconnector capacity, the variability reduction in residual demand is the dominant effect leading to lower levels of implied volatility.

\section{Empirical application to the french-german market}

In this section we apply the structural model for coupled electricity markets to the german and french market using data from 2012. As electricity spot prices we use day-ahead auction prices 

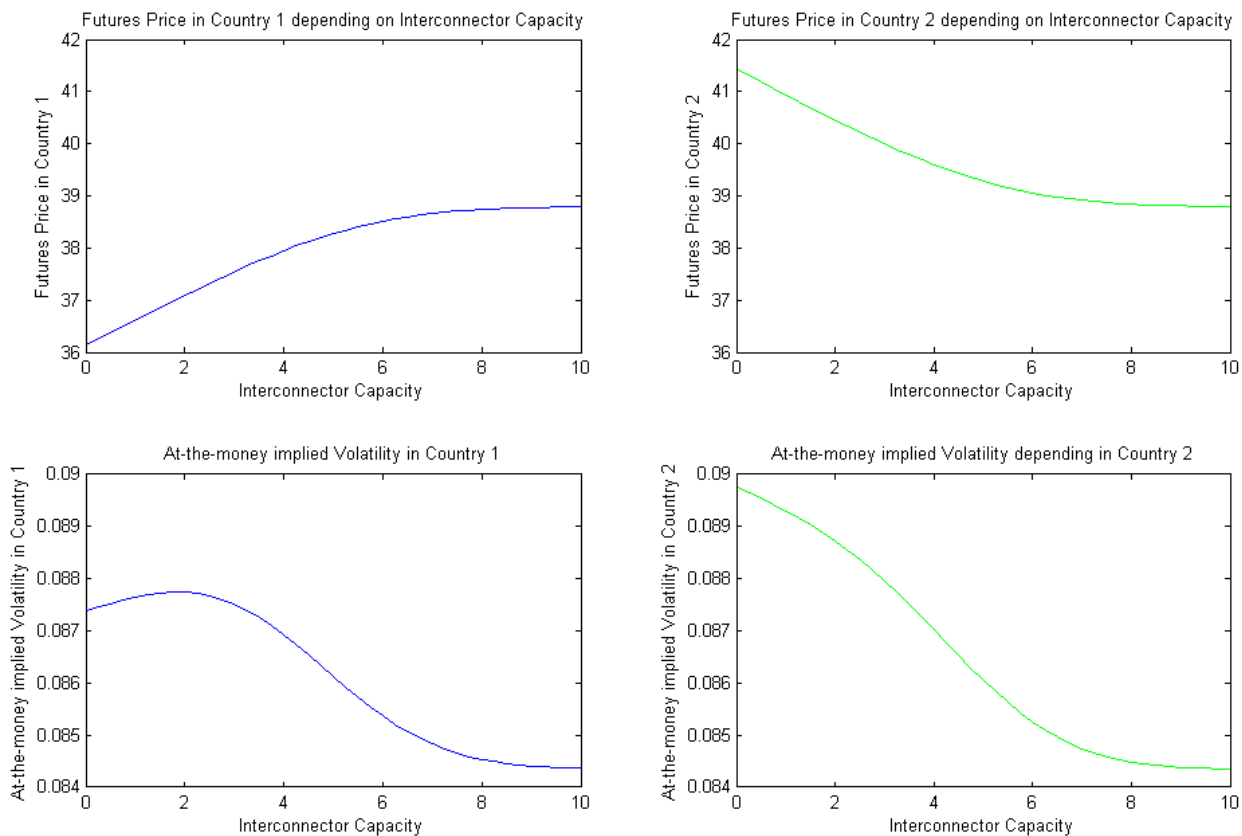

Figure 9: Model futures prices and at-the-money implied volatilities depending on interconnector capacity

from the daily auction of EPEX Spot. As fuel price we use TTF gas prices (daily closing prices) as a proxy. The implicit assumption behind this choise is that gas is the marginal fuel most of the time. Demand data was collected from the respective TSOs, installed transmission capacities are taken from the data base of entso-e. In order to be able to apply the model to the markets of Germany and France we have to assume that besides the interconnection of the two markets, there are no other links with any other electricity market, i.e. we assume the two markets to be isolated from the rest of the european electricity net.

\subsection{Market Supply Curves}

We assume the market supply curve to be given as in (18) for both markets. Denote by $\tilde{D}_{t}^{i}$ the realized expected day-ahead demand in country $i$ at time $t$ and $\tilde{P}_{t}^{i}$ the realized day-ahead price. Then, $\tilde{E}_{t}^{*}=E_{t}^{*}\left(\tilde{D}_{t}^{1}, \tilde{D}_{t}^{2}, a_{1}, a_{2}, b_{1}, b_{2}, c, \underline{E}, \bar{E}\right)$ denoteds the realized expected day-ahead exchange and $P_{t}^{*, i}\left(\tilde{D}_{t}^{i}, \tilde{E}_{t}^{*}, a_{i}, b_{i}, c, \underline{E}, \bar{E}\right)$ the model implied electricity price given the realized demand estimation for the next day. We determine the parameters of the market supply curve by minimizing the nonlinear least squares problem

$$
\sum_{i=1}^{2} \sum_{t \in \mathbb{T}}\left\|\tilde{P}_{t}^{i}-P_{t}^{*, i}\left(\tilde{D}_{t}^{i}, E_{t}^{*}, a_{i}, b_{i}, c, \underline{E}, \bar{E}\right)\right\|^{2} \rightarrow \min
$$

with respect to the parameters $a_{1}, a_{2}, b_{1}, b_{2}, c, \underline{E}, \bar{E}$. The parameters $\underline{E}, \bar{E}$ are included in this routine and not taken directly from entso-e, as the usable capacity depends on temperature. Including the parameters in the least squares problem leads to a constant weighted average of usable capacity which is used in the sequel. Table 1 summarizes the resulting values. The market supply curve in France is more convex as in Germany $\left(b_{2}>b_{1}\right)$, but sharp increases in electricity prices occur at higher demand levels $\left(a_{2}<a_{1}\right)$. The minimal electricity price of 19.98 roughly reflects the 


\begin{tabular}{ll|lr|lr}
\multicolumn{2}{c}{ Germany } & \multicolumn{2}{c}{ France } & \multicolumn{2}{c}{ Interconnector } \\
\hline Parameter & Value & Parameter & Value & Parameter & Value \\
\hline$a_{1}$ & -1.78 & $a_{2}$ & -2.73 & $\underline{E}$ & -3.5 \\
$b_{1}$ & 0.034 & $b_{2}$ & 0.047 & $\bar{E}$ & 2.8 \\
$c$ & 19.98 & $c$ & 19.98 & & \\
\hline
\end{tabular}

Table 1: Estimates of model parameters for the German and French market

marginal costs of nuclear plants. The interconnectors show to have a higher capacity from France to Germany than in the other direction which is in line with the numbers reported by entso-e. Figure 10 compares the true historical electricity prices in France and Germany with the model

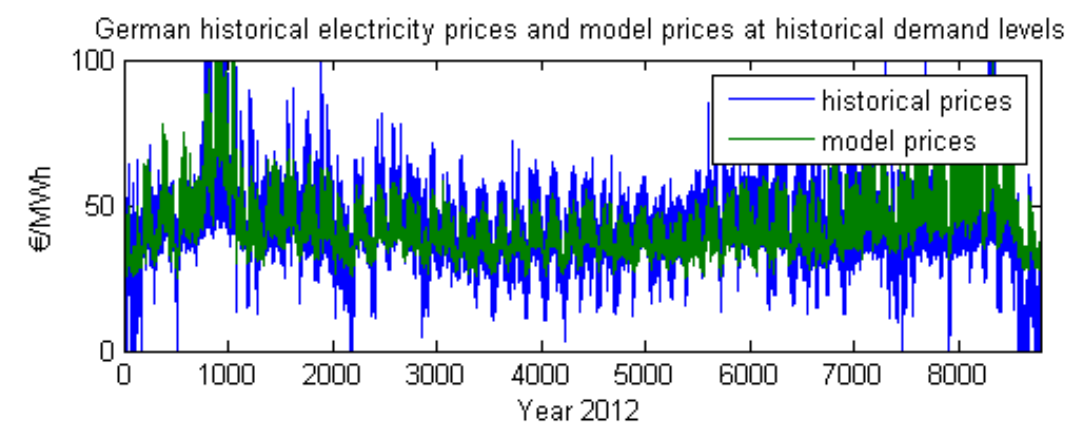

French historical electricity prices and model prices at historical demand levels

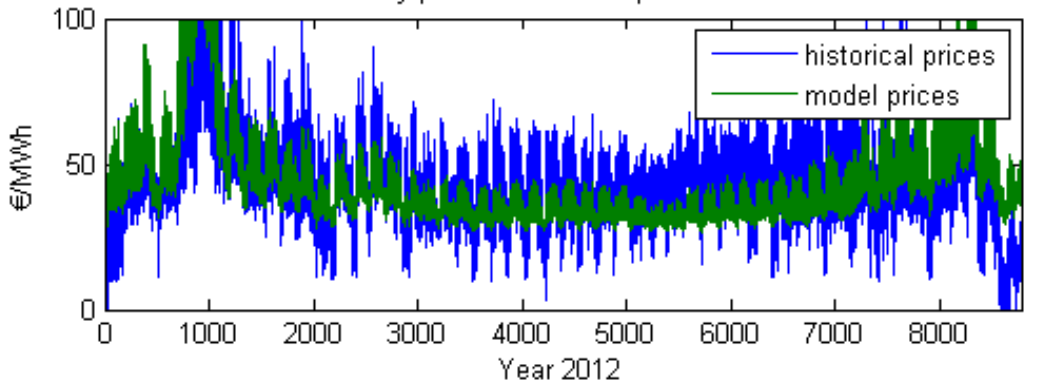

Figure 10: Historical electricity prices and model prices at historical demand levels

implied prices using the estimated parameters of the market supply curve and the true historical gas prices and demand levels. The overall level of prices is attained quite well, the variability seems to be a bit underestimated. This might be due to the fact that the true market supply curve increases superexponentially. In France, prices are underestimated during summer. This might be the case due to maintenance reasons on some of the power plants. The model does not capture variations in plant availability. Figure 11 shows the typical weekly pattern of historical prices and corresponding model prices. Although the model market supply curve is able to generate the right pattern, the variation of historical prices is higher than the variation in model prices for both countries. 

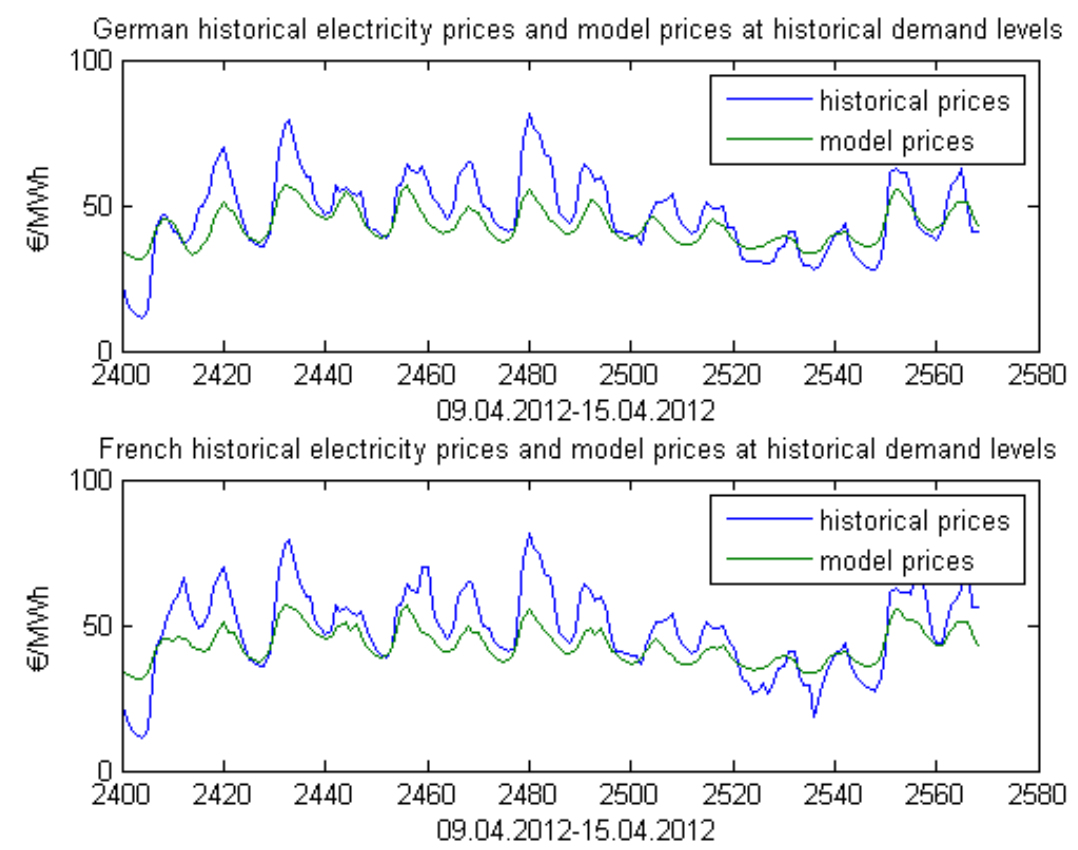

Figure 11: Weekly pattern of historical prices and model prices

\subsection{Deterministic Demand Patterns and Dynamics}

\section{Conclusion}

The two major developements in continental european electricity markets, increased infeed of renewables and increased interconnectivity of market areas, changed the structure and level of electricity prices in all affected markets. It is an ongoing challenge to develop and maintain models which are able to cope with these changes. As a single Internal Electricity Market (IEM) as it is defined as EU policy goal is not attainable in the near future, the need for models which are able to handle interconnected markets is obvious. As electricity is non-storable and load patterns as well as the avaiable power plants are well known, hybrid or structural models seem to be a good starting point to cope with market changes on the modelling side. Our research should help to understand the stylized facts wich are induced by market coupling. However, accurate pricing of individualized contracts is still very challenging. The proposed model leads to closed form futures and options prices which is a very important feature for calibration purposes and helps in keeping this model arbitrage free to any existing HPFCs for both markets. It was possible to describe two important stylized facts of coupled markets. First, introducing market coupling might lead to lower futures prices in all affected markets (even the cheapest one) due to the convexity in the market supply curve. Increasing the interconnector capacity finally leads to price convergence. Second, introducing market coupling might lead to volatility spill-over effects, meaning that options in one of the affected markets might be priced at higher implied volatilities as before even if under full market convergence, the joint volatility is lower.

Extensions to the model would include multiple markets to cope with the developement of North Western European Price Coupling (NWE) and the Price Coupling of Regions initiative (PCR). In order to get a more accurate market supply curve it would be interresting to include multiple fuels 
in the model.

\section{References}

Aid, R., Campi, L., and Langrené, N. (2013). A structural risk-neutral model for pricing and hedging power derivatives. Mathematical Finance, 23:387-438. Version: Oct 4, 2010.

Bingham, N. and Kiesel, R. (2004). Risk-Neutral Valuation. Springer.

Carmona, R. and Coulon, M. (2014). A survey of commodity markets and structural models for electricity prices. In Benth, F. E., Kholodnyi, V. A., and Laurence, P., editors, Quantitative Energy Finance, chapter 2, pages 41-84. Springer.

Carmona, R., Coulon, M., and Schwarz, D. (2013). Electricity price modeling and asset valuation: A multi-fuel structural approach. Mathematics and Financial Economics, 7:167-202.

EPEXSpot (2013). Euphemia public description. Technical report, EPEX Spot.

EPEXSpot (2014). Market coupling.

Knittel, C. R. and Roberts, M. (2005). An empirical examination of deregulated electricity prices. Energy Economics, 27(5):791-817. 